\title{
LA PERCEPCIÓN DE LOS ESTUDIANTES ACERCA DE LA PRESENCIA DE LAS TIC EN LA UNIVERSIDAD. UN ESTUDIO EN EL ÁMBITO DE LA INGENIERÍA EN COLOMBIA
}

\section{STUDENTS' PERCEPTION ABOUT THE USE OF ICTS AT THE UNIVERSITY. AN OVERVIEW FROM THREE ENGINEERING FACULTIES IN COLOMBIA}

\author{
Juan González Martínez; juan.gonzalez@udg.edu \\ Universitat de Girona, España \\ Fernando Martínez Rodríguez; fmartinezr@udistrital.edu.co \\ Universidad Distrital Francisco José de Caldas, Colombia
}

\section{RESUMEN}

La penetración de las TIC en la docencia universitaria sigue siendo superficial, y en ocasiones lejana de un verdadero cambio pedagógico. En la descripción de los diferentes contextos universitarios habitualmente se tiene en cuenta la percepción del profesorado universitario acerca de su propio uso de la tecnología, pero no se atiende a la visión del alumnado. Esta investigación analiza la percepción del alumnado sobre el uso de las TIC por parte de sus docentes de Ingeniería en Colombia por medio de una encuesta $(n=515)$. Los informantes confirman que las TIC están presentes de en la docencia, pero ligadas a usos tradicionales y con un impacto positivo pero superficial en su aprendizaje. En parte, ello se debe a que las tecnologías más usadas son aquellas que los docentes usan en otros contextos de su desarrollo profesional, y no aquellas que podrían resultar más provechosas para el aprendizaje del alumno. Dicho impacto es percibido mayor cuando las TIC se usan con más frecuencia.

Palabras clave: TIC, percepción, estudiantes, universidad, Colombia.

\section{ABSTRACT}

The penetration of ICTs at the university remains superficial, and sometimes far from a true pedagogical change. In the description of the different university contexts we usually take into account the university professors' perception about their own technological use, but we do not attend to students' point of view. This research specifically analyzes the students' perception of the use of ICT by their teachers in three Engineering faculties in Colombia through a survey $(n=515)$. Informants confirm that ICTs are regularly present when their professors teach them, but linked to 
traditional uses and with a positive but superficial impact on their learning. In part, this is due to the fact that the most used technologies are those that teachers seem to use in other contexts of their professional development, not those that could be more profitable for the students' learning. Finally, such impact is perceived to be greater when ICTs are used more frequently.

Keywords: ICT, perception, students, university, Colombia.

\section{INTRODUCCIÓN}

Es un lugar común, pero no por ello menos cierto, iniciar cualquier reflexión sobre la presencia de las TIC en los procesos educativos del siglo XXI con la constatación de la que Sociedad del Conocimiento conlleva indefectiblemente el consumo general y masivo de las Tecnologías de la Información y la Comunicación (TIC, en lo sucesivo). Y ello aplica a la ciudadanía por completo, y a la industria del sector en particular. Sin duda, las TIC están presentes en gran parte de nuestras actividades personales y profesionales actuales, de tal forma que muchos jóvenes difícilmente conciben muchas de las facetas de su vida sin su dimensión digital. Como consecuencia de ello, las TIC suponen también un sector estratégico de importancia innegable. Por ejemplo, como señala el informe ministerial colombiano, el PIB del sector TIC y de las telecomunicaciones ha crecido en los últimos años y se sitúa en torno al $5 \%$ para el 2015, lo que indica el potencial del sector (Sánchez, Hoyos, \& Mejía, 2015).

Es imposible no reconocer las consecuencias que tiene todo esto en las universidades de todo el mundo, que en ningún caso pueden mantenerse al margen. Esta intuición contrasta con la obviedad de que las universidades latinoamericanas, en general, y las colombianas en concreto han realizado muchos esfuerzos por seguir la senda de la vanguardia tecnológica, como no podría ser de otro modo. Y así lo reconoce de modo inequívoco gran parte de la bibliografía especializada del ámbito. Las universidades, en efecto, dedican gran parte de sus presupuestos a la actualización de sus recursos materiales en lo que tiene que ver con la adquisición y con la actualización de las tecnologías digitales (y no es algo solo del pasado, sino del presente y a buen seguro del futuro más inmediato) (Guzmán Flores, 2008; Torres Gastelú, 2011; Zenteno Anzira \& Mortera, 2011), y también a la capacitación y alfabetización tecnológica de sus recursos humanos para que puedan hacer frente al reto de la inclusión de las TIC en la docencia (López de la Madrid, 2007; López de la Madrid \& Chávez Espinoza, 2013). Con todo, no siempre las metas alcanzadas son las deseadas (ni por los alumnos, ni por las instituciones en su conjunto), y en las universidades latinoamericanas se documenta un general uso de las TIC bastante residual (Guzmán Flores, 2008; Torres Gastelú, 2011; Valerio Mateos \& Paredes Labra, 2008; Zenteno Anzira \& Mortera, 2011).

Precisamos, por ende, planes institucionales (Carrasco, 2010; Prendes Espinosa \& Castañeda Quintero, 2010; Rosario Noguera \& Vásquez Melo, 2012; Suárez Rodríguez, Almerich, Gargallo López, \& Aliaga, 2010); estrategias integrales para cada universidad que, tras el exhaustivo diagnóstico particular, tracen planes de formación que permitan apropiarse a los docentes apropiarse de las TIC para integrarlas de forma efectiva en los procesos de enseñanza-aprendizaje (Cela-Ranilla, Esteve, Esteve Mon, 
González Martínez, \& Gisbert Cervera, 2015; Esteve Mon, Larraz Rada, Gisbert Cervera, \& Espuny Vidal, 2011). Únicamente con estos planes estratégicos podremos revertir los modelos pedagógicos actuales de las instituciones de formación superior para enfrentarnos de modo efectivo a los retos del siglo XxI.

Ante toda esta necesidad de diseñar planes institucionales que permitan impulsar de forma efectiva y productiva la inclusión de las TIC en la docencia universitaria en general y en el ámbito de ingeniería en particular, como decíamos, una de las primeras acciones será el diagnóstico. Y en él, la percepción de los estudiantes ante lo que está sucediendo también puede ser relevante como parte de esa necesaria radiografía inicial (Maquilón Sánchez Javier J., Mirete Ruiz, García Sánchez, \& Hernández Pina, 2013; Morales Capilla, Trujillo Torres, \& Raso Sánchez, 2015). Por ello, nuestro objetivo en este artículo es analizar las percepciones del alumnado del área de ingeniería de tres universidades colombianas sobre el uso de las TIC por parte de sus profesores, a fin de complementar otros análisis sobre el uso de las TIC por parte de ese mismo colectivo (Martínez Rodríguez \& González Martínez, 2015).

\section{MARCO CONCEPTUAL}

Como decíamos al inicio, sin duda, la inclusión de las TIC en la formación universitaria supone un reto ineludible para las instituciones de formación superior que liga de modo directo con la formación de los docentes, que no siempre están formados ni experimentados al respecto (Bates \& Sangrà, 2012). Las universidades colombianas, desde luego, se han ocupado de ello en buena medida, y así, por ejemplo, podemos documentar el caso de las Facultades de Ingeniería, en las que el acopio de recursos tecnológicos ha sido importante, del mismo modo que la oferta de formación se ha ido generalizando con el paso de los años, hasta el punto de que gran parte del profesorado reconoce haber participado en acciones de capacitación al respecto (Martínez Rodríguez \& González Martínez, 2015). El uso de las TIC en los procesos académicos, escaso y superficial, pues, no está fundamentado en una baja disponibilidad de recursos TIC en los salones de clase, ni tampoco en una falta absoluta de formación para que el profesorado pueda sentirse capacitado en su manejo técnico. Y no es eso, en absoluto, lo que recomiendan las instituciones mundiales de referencia. Por ejemplo, la UNESCO (2008) recomienda que la formación universitaria incluya las TIC para poder garantizar la adquisición de las competencias, contribuir en la búsqueda y en la evaluación de la información, ayudar en la toma de decisiones, mejorar la comunicación y la interacción, la difusión del conocimiento; y, en definitiva, la productividad de todos los profesionales. Es evidente cuán difícil es imaginarse una universidad de nuestro tiempo que pueda ofrecer todo ello sin el concurso eficaz de las TIC.

No obstante, la incidencia de las TIC en la universidad latinoamericana es baja (Zenteno Anzira \& Mortera, 2011), y eso tiene consecuencias importantes en la formación de los universitarios. Por ejemplo, en su análisis de los docentes mexicanos, se refiere que apenas un 39 \% utiliza alguna tecnología en su docencia (Vera Noriera, Torres Moran, \& Martínez García, 2014); sin embargo, en estos casos, a menudo lo que realmente encontramos es la conversión de estrategias tradicionales sin cambio 
didáctico asociado (Valerio Mateos \& Paredes Labra, 2008); pero no se producen incursiones en la virtualización, ni ampliar los usos tecnológicos a otros contextos de acción universitarios como la gestión o la tutoría, que también se pueden beneficiar al respecto. $Y$ eso, como contrapartida de las importantes inversiones en infraestructuras llevadas a cabo, indica a todas luces un cierto desaprovechamiento (Espinosa, 2010; Prendes Espinosa \& Castañeda Quintero, 2010).

En definitiva, la apropiación de las TIC por parte del profesorado y su uso general y productivo en la docencia universitaria aún no se puede dar por consolidada, por lo que no podemos considerar aún que en el conjunto de las universidades colombianas se da un uso pertinente de la tecnología educativa ni de un aprovechamiento didáctico real (UNESCO, 2005). Como ejemplo, Zubieta, Bautista y Quijano (2012) indican que a pesar de los altos niveles de intención de uso de las TIC por parte de los docentes, la frecuencia en su uso es limitada, y lo son también os ambientes web utilizado. Y, más aún, según Guzmán, García, Espuny y Chaparro (2011), señalan que la utilización de las TIC por los docentes se centra en la Web 1.0, mientras que los estudiantes tienen a preferir la Web 2.0.

Hasta aquí hemos venido hablando de como las TIC han ido penetrando con desigual fortuna en las universidades, y de cómo gran parte de la atención se ha venido centrando en la lógica necesidad de que el profesorado se forme al respecto y se empodere para aprovechar todo el potencial educativo de las TIC en la docencia superior (Díaz-Barriga Arceo, 2010; Torres Gastelú, 2011). En este sentido, es oportuno reconocer que parte del diagnóstico general que se ofrece dibuja un escenario en el cual el conjunto del profesorado usa las TIC en gran medida para preparar sus sesiones presenciales o sus materiales educativos, pero no para el proceso de aprendizaje del alumnado, que sigue siendo tradicional en exceso (Comisión Europea, 2013). Sin embargo, si finalmente de lo que se trata es de mejorar el aprendizaje del alumnado, y no solo el ejercicio profesional del profesorado, deberemos prestarles atención especial a ellos, por cuanto su visión puede colaborar con este diagnóstico general y confirmar si realmente la incorporación de las TIC ha sido realmente productiva (Rosario Noguera \& Vásquez Melo, 2012; Valerio Mateos \& Paredes Labra, 2008). En este sentido, Del Moral, Villalustre y Neira (2016) señalan que el conjunto de la comunidad universitaria confirma un impacto positivo de su inclusión en el proceso de aprendizaje, si bien todos los colectivos (y especialmente el profesorado) debería mejorar su formación al respecto.

Como consecuencia de ello, Claro (2010) indica que al observar los diferentes usos de las TIC en la docencia, y su impacto en el aprendizaje, el alumnado echa en falta más personalización y más colaboración tanto en las estrategias diseñadas como en las herramientas TIC usadas; $y$, por supuesto, aboga por que se desdibuje el límite aún demasiado marcado entre lo formal y lo informal en especial por lo que se refiere a las TIC (Comisión Europea, 2013). Y, por lo que respecta a la percepción que tienen del conocimiento y del uso tecnológico del profesorado, es habitual diagnosticar que se usan en mayor medida los recursos de gestión y tratamiento de información, y en menor proporción aquellos que tienen que ver con la creación de recursos didácticos o con la interacción o la dimensión social profesor-alumno (Mirete Ruiz, 2016).

En cualquier caso, las percepciones de los estudiantes al respecto son siempre 
positivas, y es común recabar información positiva acerca de las actitudes del alumnado hacia al uso que los docentes practican de las TIC en clase o en los espacios virtuales, pues el alumnado es consciente de su importancia en términos de impacto y de las innumerables posibilidades que ofrecen (Morales Capilla et al., 2015). Es más, a pesar de que su uso pueda llegar a ser residual, los estudiantes universitarios confirman la pertinencia general del uso TIC del profesorado, y en especial su impacto en el propio aprendizaje (Maquilón Sánchez Javier J. et al., 2013). Y eso es común a pesar de que los propios estudiantes puedan pertenecer o no a cualquiera de los perfiles según el uso deseado frente a las TIC, que a su vez son los que menos las usan en la gestión de su propio aprendizaje (Santamaría, SanMartín, \& López, 2014). Y, como reconocen estos tres autores, es siempre importante prestar atención a la percepción del alumnado a fin de contribuir un poco más, y mejor, a su aprendizaje por medio de las TIC.

\section{CONTEXTO DE LA INVESTIGACIÓN}

Nuestra investigación tiene por marco general el proyecto titulado Referentes pedagógicos para el uso y apropiación de las Tecnologías de la Información y Comunicación (TIC) al interior de los procesos educativos planeados y desarrollados por los docentes de educación superior, dentro del cual supondría la fase diagnóstica. Dicho proyecto tiene por objetivo general formular y validar referentes pedagógicos que permitan orientar a instituciones y docentes de educación superior, colombianos en primera instancia, en el adecuado uso y apropiación de las TIC al interior de los procesos educativos orientados por ellos. Este proyecto tiene como contexto concreto las facultades Ingeniería de las diferentes universidades del país. Y toma como referencia tres instituciones en concreto:

- Universidad Distrital Francisco José de Caldas (en adelante, UD). Esta es la universidad pública de Bogotá, con más de 27.000 estudiantes, 45 programas de pregrado y 34 programas de postgrado.

- Universidad de la Salle (en adelante, UniSalle). Se trata de una universidad privada con acreditación de alta calidad; cuenta con más de 13.000 estudiantes, 23 programas de pregrado y 24 programas de postgrado.

- Escuela Tecnológica Instituto Técnico Central (en adelante, ETITC). Es una institución pública de educación superior con sede en Bogotá; en la actualidad cuenta únicamente con programas de ingeniería bajo la modalidad de ciclos propedéuticos. Tiene más de 4.000 estudiantes; 15 programas de pregrado contados entre los ciclos: técnico, tecnológico y profesional.

Como en el resto de Latinoamérica, las universidades colombianas han realizado innumerables esfuerzos por la modernización de sus instalaciones, de sus recursos y de sus procesos, y de ello no escapa la incorporación de las TIC. Sin embargo, el diagnóstico general no es excesivamente positivo, en la medida en que se documenta que en general parece predominar el uso tecnológico en la docencia que ya tiene arraigo en las demás facetas de la vida académica del docente, los docentes suelen seguir la propuesta tecnológica institucional sin excesivas incorporaciones (web de la 
universidad, catálogos de las bibliotecas) y el uso de recursos destinados al trabajo colaborativo en clase es considerablemente bajo (Said-Hung et al., 2015). Así lo reconocía también la propia UNESCO (ECOSOC, 2011), en su estudio sobre la situación de las TIC en toda la región.

Como consecuencia de ello, la principal problemática con la que se encuentra la universidad colombiana frente al reto de las TIC tiene que ver precisamente con este bajo uso de las TIC en nuevas estrategias didácticas, con un uso que no atiende a referentes pedagógicos que supongan una auténtica renovación, y con una penetración de las TIC que en definitiva no produce cambios significativos (PolaníaMuñoz, Córdoba-Beltrán, \& López de Parra, 2015), a pesar de lo exitosas que se han mostrado las prácticas de liderazgo TIC en las instituciones en que se han producido (Cifuentes-Álvarez \& Vanderlinde, 2015). Y de ahí que en gran medida hayan sido el propio gobierno colombiano quien se haya comprometido con la cuestión (MEN, 2008).

En el caso concreto que nos ocupa, las Facultades de Ingeniería de las universidades colombianas, los docentes no escapan a este diagnóstico general, por bien que no sea tan negativo. El profesorado usa las TIC, pero es evidente también la necesidad de orientar estas prácticas educativas hacia un uso más adecuado y productivo desde el punto didáctico y pedagógico (Martínez Rodríguez \& González Martínez, 2015). Así, por ejemplo, puede verse como en líneas generales el grueso del profesorado sondeado en aquel estudio confirmaba usar las TIC de forma habitual (Gráfico 1):

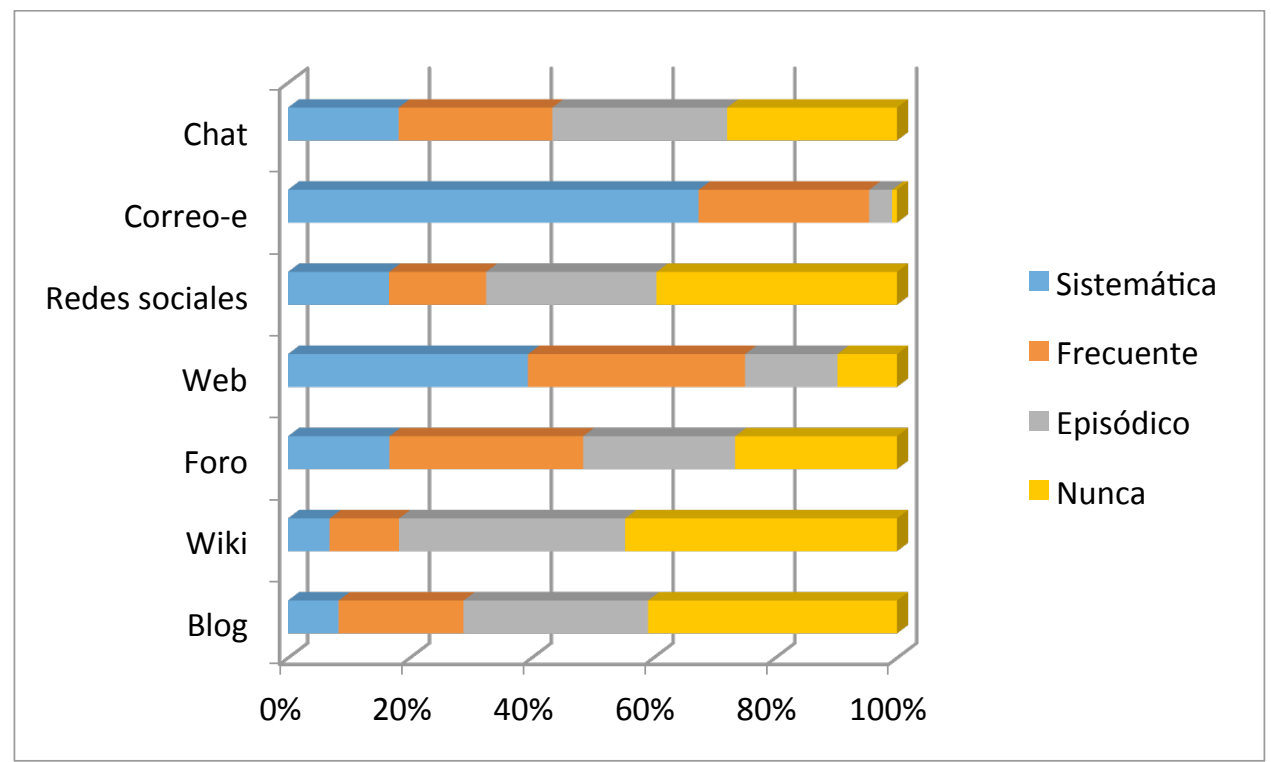

Gráfico 1. Uso de las TIC en la práctica docente por parte del profesorado de Ingeniería, según Rodríguez y González (2015).

En este gráfico, se aprecia claramente cómo determinadas opciones tecnológicas ligadas a la Web 1.0 son recurrentes, especialmente aquellas que tienen que ver con recursos tecnológicos ya usados por el profesorado en otras facetas personales o profesionales ajenas a la docencia. Y, por otro lado, también se confirma que las TIC están más presentes en las tareas de planificación y gestión del profesorado que en su propia docencia, como podemos comprobar en la Gráfica 2: 


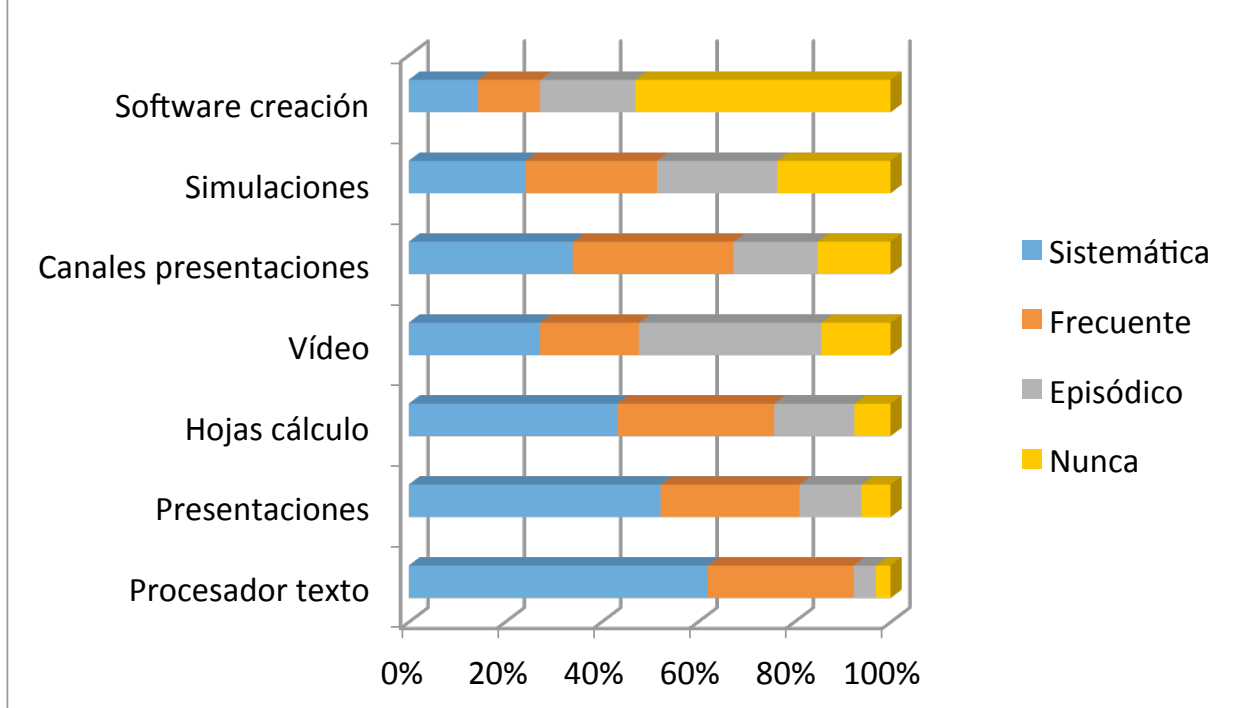

Gráfico 2. Uso de las TIC en la planificación y en la preparación de la docencia por parte del profesorado de Ingeniería, según Rodríguez y González (2015).

\section{MÉTODOS}

Como se deduce del objetivo de la investigación, presentamos una investigación descriptiva cuantitativa llevada a cabo por medio de un cuestionario on-line (Bisquerra Alzina, 2004). La herramienta que hemos utilizado es el cuestionario autoadministrado creado y validado por expertos para esta investigación. En dicho cuestionario, que se encabeza con las orientaciones iniciales y el debido consentimiento informado, contiene las siguientes secciones: (1) biodatos, (2) percepción general sobre el uso de las TIC por parte de sus docentes, (3) percepción detallada sobre el uso de determinados recursos TIC por parte de sus docentes. En cada una de las secciones 2 y 3 , además, se preguntaba a los informantes sobre el impacto de esos usos en el propio aprendizaje (en una escala gradiente de Likert de 4 puntos) y sobre la frecuencia con que esos usos eran practicados.

En cuanto al proceso de creación y validación del instrumento, inicialmente el cuestionario fue socializado a pares, para analizar la pertinencia y alcance del mismo; una vez aprobado por los pares, el instrumento fue validado, aplicando una prueba piloto a 7 estudiantes de pregrado para evaluar la coherencia, la comprensión (claridad y redacción) de las preguntas. Por otro lado, se calculó la consistencia interna del mismo a través del test de Alfa de Cronbach, y se obtuvo un puntaje de 0.821 . Este mismo cálculo se hizo a partir de las 515 encuestas recabadas, en donde se obtuvo un valor de 0.845 , con lo cual se considera altamente confiable (George \& Mallery, 1995).

En cuanto al tamaño de la muestra en relación con la población analizada, diremos que Para obtener el tamaño de la muestra en cada universidad, se seleccionó en cada una de ellas un $95 \%$ de nivel de confianza y un margen de error entre el $6 \%$ y el $11,8 \%$ (Vivanco, 2005). Bajo estos parámetros, el tamaño de la muestra óptimo calculado para cada una de las 3 universidades escogidas fue el siguiente: 
- Universidad Distrital: Tamaño de la muestra: 221; Margen de error: 6,3\%

- Universidad de la Salle: Tamaño de la muestra: 221; Margen de error: 6,4\%

- Escuela Tecnológica Instituto Técnico Central: Tamaño de la muestra: 66; Margen de error: $11,8 \%$

\section{RESULTADOS}

Como decíamos al inicio, la encuesta se practicó sobre la muestra de 515 informantes que decidieron responder el cuestionario que se les ofreció. De ellos, un 61,4\% eran hombres, y una 38,6 \% eran mujeres. En cuanto a las franjas de edad, un 0,6\% tenía menos de 16 años, un 14,8 \% se situaba entre los 17 y los 19 años, un 35,8 \% tenía entre los 20 y los 22 años, y un $48.8 \%$ superaba los 22 años.

Por universidades, el 42,9 \% de la muestra procedía de la UD, el mismo 42,9 \% procedía de UniSalle y un 14,2 \% procedía del ITITC. Finalmente, en relación con el semestre que cursaban en su programa de estudios, un $18,1 \%$ se situaba en la fase inicial de sus estudios (semestres 1 a 4), un 24,8\% se situaba en su parte central (semestres 5 a 8) y un 57,1\% se situaba en la parte final (semestres 9 a 12).

Si vamos ya a los datos recabados, observaremos una primera imagen positiva. Según se recoge en la Tabla 1, los alumnos perciben que el uso de las TIC en general en el aula es alto, con un $76,8 \%$ de las respuestas que indican que los profesores las usan en el aula y un $69,58 \%$ que afirman que los profesores son proactivos al sugerir el uso de las TIC (no solo las usan, sino que promueven su uso, lo recomiendan).

\begin{tabular}{|l|l|l|}
\cline { 2 - 3 } \multicolumn{1}{c|}{} & Sí & No \\
\hline Uso de las TIC en el aula & 76,8 & 23,2 \\
\hline Proactividad del profesorado con las TIC & 69,5 & 30,5 \\
\hline
\end{tabular}

Tabla 1. Uso general de las TIC por parte del profesorado

Valoración diferente merece el análisis del uso detallado de los diferentes recursos tecnológicos por los que sondeábamos al alumnado (Gráfica 3). A este respecto, es claro que el uso percibido es muy desigual. Encontramos algunos recursos que son ampliamente utilizados, como las aulas virtuales, la web, el correo electrónico, los documentos digitales o el software específico; por el contrario, otros están poco presentes a tenor de lo que nos indican los informantes, como los blogs, las wikis, los foros, las redes sociales o el vídeo. Si analizamos esto con un poco de detalle, veremos que puede responder a dos patrones. Por un lado, los más usados son aquellos que a buen seguro tienen más uso en las restantes facetas de la acción profesional del profesorado (planificación de las clases, gestión, investigación); y, por el otro, gran parte de los menos utilizados son aquellos que tienen más que ver con la 2.0, esto es, con la reversión de los estáticos roles de la emisión y la recepción o, si se quiere, con la colaboración por medio de la red. 


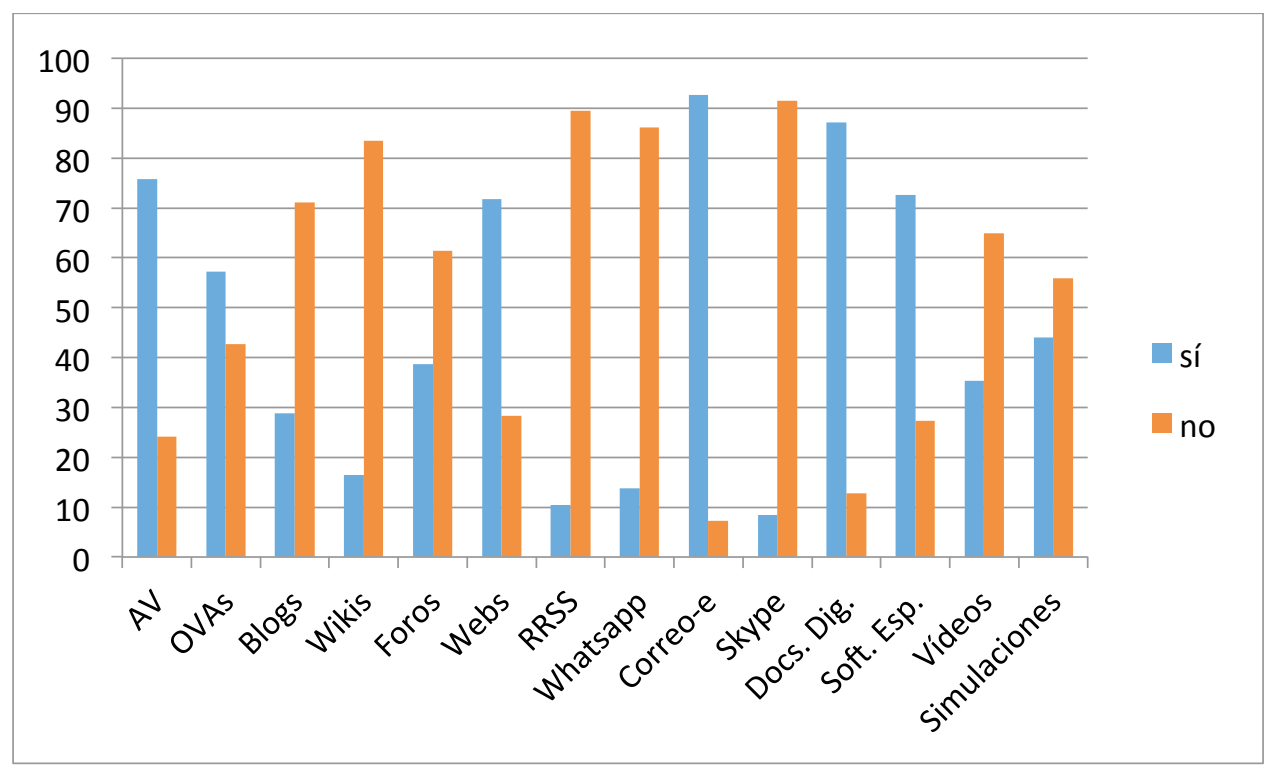

Gráfica 3. Uso detallado de las TIC por parte del profesorado (en \%).

Otra de las cuestiones que nos puede resultar interesante en este análisis de la percepción del profesorado tiene que ver con la secuencia completa entre la presencia de las TIC en clase o la proactividad del profesorado al respecto, la frecuencia con que esto ocurre y la calidad que percibe el alumnado que esto aporta a su aprendizaje, su impacto en el aprendizaje (Tabla 2). Si tanto la calidad como la frecuencia de uso se expresan en escala de 4 puntos, veremos que la valoración es positiva (pero no marcadamente positiva) en relación con el impacto positivo que los informantes consideran que tiene tanto este uso de las TIC en la docencia por parte del profesorado, como relativamente baja es la frecuencia con que se usan. Esto es, los profesores usan y recomiendan las TIC, pero no de manera sistemática cuando lo hacen; $y$, sea como sea, cuando esto ocurre, la percepción del alumnado es que ello no es marcadamente positivo.

\begin{tabular}{|l|l|l|l|}
\cline { 2 - 4 } \multicolumn{1}{c|}{} & \% uso & Impacto & Frec. uso \\
\hline TIC en clase & 76.8 & 2,644 & 2.95 \\
\hline Proactividad TIC & 69.5 & 2,677 & 2.64 \\
\hline
\end{tabular}

Tabla 2. Valoración general del alumnado del uso de las TIC por parte del profesorado

Más positiva es, sin embargo, la valoración de estos mismos datos cruzados, detallados por recursos. En este caso, en la Tabla 3 vemos toda la gama que encontrábamos en líneas generales en la Gráfica 3, con sus correspondientes percepciones en cuanto la calidad y a frecuencia de uso. Ahí se puede observar que los informantes valoran más positivamente el impacto de los recursos en su aprendizaje en concreto, con independencia de que se usen con más o menos frecuencia. $Y$, eso sí, se aprecia también que los más usados también son los que se usan con mayor frecuencia. 


\begin{tabular}{|l|l|l|l|}
\cline { 2 - 4 } \multicolumn{1}{c|}{} & \% uso & Impacto & Frec. uso \\
\hline Correo electrónico & 92,7 & 3,0 & 2,7 \\
\hline Docs. digitales & 87,2 & 3,1 & 2,7 \\
\hline Aulas Virtuales & 75,8 & 2,7 & 2,7 \\
\hline Software esp. & 72,6 & 3,0 & 2,8 \\
\hline Sitios Web & 71,7 & 2,9 & 2,3 \\
\hline OVAs & 57,3 & 2,8 & 2,7 \\
\hline Simulaciones & 44,1 & 3,0 & 2,3 \\
\hline Foros & 38,6 & 2,8 & 2,1 \\
\hline Vídeo & 35,4 & 3,0 & 2,1 \\
\hline Blogs & 28,9 & 2,8 & 2,4 \\
\hline Wikis & 16,5 & 2,8 & 2,5 \\
\hline Whatsapp & 13,8 & 2,6 & 2,5 \\
\hline Redes sociales & 10,5 & 2,7 & 2,5 \\
\hline Videoconferencia & 8,5 & 3,0 & 1,9 \\
\hline
\end{tabular}

Tabla 3. Valoración detallada del alumnado del uso de las TIC por parte del profesorado

Al analizar el impacto que el alumnado percibe en el aprendizaje por el uso de las TIC en función del momento de los estudios en que se encuentra (Tabla 4), vemos que hay diferencias significativas entre los dos extremos (al final de los estudios el impacto percibido en el aprendizaje es menor) y entre el inicio y el medio de los estudios; entre los semestres mediales y los finales la media aumenta, pero no de forma que se pueda considerar significativa, a tenor de los resultados.

\begin{tabular}{|l|l|l|l|}
\hline \multirow{4}{*}{ (Semestre (I) } & Semestre (J) & $\begin{array}{l}\text { Diferencia de } \\
\text { medias (I-J) }\end{array}$ & Sig. \\
\hline \multirow{2}{*}{ Inicio } & Medio &, $3068^{*}$ &, 028 \\
\cline { 2 - 4 } & Final &, $4849^{*}$ &, 000 \\
\hline \multirow{2}{*}{ Medio } & Inicio &,$- 3068^{*}$ &, 028 \\
\cline { 2 - 4 } & Final &, 1781 &, 154 \\
\hline \multirow{2}{*}{ Final } & Inicio &,$- 4849^{*}$ &, 000 \\
\cline { 2 - 4 } & Medio &,- 1781 &, 154 \\
\hline
\end{tabular}

Tabla 4. Diferencias en la valoración del impacto de las TIC en función del avance de los estudios por parte del alumnado.

Por otro lado, no documentamos diferencias significativas entre las tres universidades, que comparten por tanto este diagnóstico general, ni por géneros o por grupos de edad. En cuanto a los análisis de covarianza, no se aprecia la incidencia relevante de ninguno de los ítems cuestionados, ni para la configuración de la percepción general de uso ni para la de calidad o impacto en el propio aprendizaje, por lo que con los datos que tenemos no podemos profundizar en un patrón claro a este respecto.

Finalmente, al analizar la correlación entre las diferentes variables, destacamos la que se produce (positiva y significativa) entre la percepción del impacto en el propio aprendizaje y la frecuencia de uso en clase (Tabla 5), que nos indica que los alumnos tienen la percepción (lógica, por otra parte) que las tecnologías más frecuentemente 
presentes en su proceso de aprendizaje son las que mejor impactan en él en términos de construcción del conocimiento.

\begin{tabular}{|l|l|l|}
\hline \multicolumn{2}{|c|}{} & $\begin{array}{l}\text { Frecuenci } \\
\text { a }\end{array}$ \\
\hline \multirow{3}{*}{ Impacto } & $\begin{array}{l}\text { Correlación de } \\
\text { Pearson }\end{array}$ &, $460^{* *}$ \\
\cline { 2 - 3 } & Sig. (bilateral) &, 000 \\
\hline
\end{tabular}

Tabla 5. Correlación entre las medias de Impacto en el aprendizaje y Frecuencia de uso percibido.

\section{DISCUSIÓN Y CONCLUSIONES}

Como se desprende del análisis anterior, la percepción de los estudiantes complementa y confirma aquello que ya conocíamos por anteriores análisis de los usos TIC del profesorado, por un lado; y por la revisión de la literatura de lo que acontece en otros contextos universitarios vecinos. En ese sentido, yendo de lo general a lo particular, si retomamos las conclusiones de Rodríguez y González (2015), veremos que, en efecto, la generalización del uso de las TIC en la docencia universitaria de las Facultades de Ingeniería colombianas es alta: la tecnología educativa no solo es referida como habitual por los docentes, sino que también el alumnado tiene la sensación de que las TIC forman parte de la cotidianeidad de su vida escolar en la universidad. Con todo, el análisis detallado de esas mismas percepciones indica que esa generalización es superficial, tradicional; y que va ligada más al envoltorio de los procedimientos que al cambio profundo de las prácticas educativas. Es algo que diferentes instituciones vienen censurando desde hace ya unos cuantos años (Comisión Europea, 2013; UNESCO, 2005) y que, sin embargo, los estudiantes nos permiten confirmar que aún ocurre.

Así, por un lado nos encontramos que los datos generales son positivos, pero por el otro, en el detalle, la percepción de los estudiantes indica que la frecuencia de uso de las TIC (cuando se usan) no es especialmente sistemática y, como consecuencia de ello, el impacto en su aprendizaje no es tan provechoso como podría ser.

Quizá parte de las causas de eso tenga que ver con el hecho de que los usos tecnológicos que el alumnado percibe tienen que ver con prácticas que ligan más con la Web 1.0 (inidireccionales, no interactivas, que conciben al destinatario como un ente pasivo y no activo), y no tanto con la Web 2.0 (justo lo contrario), que es lo que los alumnos esperarían (Guzmán Flores et al., 2011). Porque la percepción general es buena, en efecto, y en ese sentido coincide con lo que se indica habitualmente en relación con cómo el alumnado recibe la tecnología en el contexto universitario (Almerich, Orellana, \& Díaz-García, 2015; Maquilón Sánchez Javier J. et al., 2013); pero acaso no lo sea tanto como debería precisamente porque las TIC con presencia en el aprendizaje del alumnado no están pensadas para él, sino que manan de las prácticas de gestión o de investigación. Esto es, que el profesorado parece más tendente a usar aquello que ya conoce por otras facetas personales y profesionales que a innovar en función de qué puede mejorar la experiencia de aprendizaje del alumnado (Martínez Rodríguez \& González Martínez, 2015). 
¿Cuál es la solución a todo esto? Sin duda, el diseño y la aplicación de planes institucionales (Prendes Espinosa \& Castañeda Quintero, 2010; Rosario Noguera \& Vásquez Melo, 2012; Suárez Rodríguez et al., 2010) que permitan al profesorado combatir sus renuencias a la incorporación real, efectiva e innovadora de las TIC (Cabero, 2003). Y, por supuesto, que dichos planes partan de diagnósticos como el actual, que no solo tienen en cuenta el conocimiento general, sino el análisis detallado de la realidad universitaria para la cual se diseñan dichos planes estratégicos.

\section{REFERENCIAS}

ALMERICH, G., ORELLANA, N., \& DÍAZ-GARCÍA, I. (2015). Las competencias en TIC en el profesorado en formación y su relación con las creencias pedagógicas, la autoeficacia y la percepción del impacto de las TIC en la educación. In AIDIPE (Ed.), Investigar con y para la sociedad (pp. 589-597). Cádiz (España): Bubok.

BATES, A. W., \& SANGRÀ, A. (2012). La gestión de la tecnología en la educación superior. Barcelona: Octaedro ICE-UB.

BISQUERRA ALZINA, R. (2004). Metodología de la Investigación Educativa (2. edició). Madrid: La Muralla.

CABERO, J. (2003). Incidentes críticos para la incorporación de las TICs a la universidad. In EDUTEC (Ed.), Edutec Venezuela. Caracas: Universidad Central de Venezuela.

CARRASCO, S. (2010). Paradojas y dilemas de las universidades iberoamericanas ante la sociedad del conocimiento, by Adriana Gewerc Barujer (coord.) (reseña). RUSC. Universities and Knowledge Society Journal, $7(1)$. http://doi.org/10.7238/rusc.v7i1.662

CELA-RANILLA, J., ESTEVE, V., ESTEVE MON, F. M., GONZÁLEZ MARTÍNEZ, J., \& GISBERT CERVERA, M. (2015). El docente en la sociedad digital: una propuesta basada en la pedagogía transformativa y en la tecnología avanzada. Profesorado. Revista de Currículum Y Formación Del Profesorado, [En prensa.

CIFUENTES-ÁlVAREZ, G., \& VANDERLINDE, R. (2015). Liderazgo de las TIC en educación superior: estudio de caso múltiple en Colombia. Comunicar, 23(45), 133-142. http://doi.org/10.3916/C45-2015-14

CLARO, M. (2010). Impacto de las TIC en los aprendizajes de los estudiantes. Santiago de Chile: CEPAL.

COMISIÓN EUROPEA, C. (2013). Docencia y aprendizaje innovadores para todos a través de nuevas tecnologías y recursos educativos abiertos. Bruselas.

DEL MORAL, M. E., VILLALUSTRE, L., \& NEIRA, M. R. (2016). Oportunidades de las TIC para la innovación educativa en las escuelas rurales de Asturias. Aula Abierta, 42(44), 7-14. http://doi.org/10.1016/j.aula.2014.12.001

DÍAZ-BARRIGA ARCEO, F. (2010). Los profesores ante las innovaciones curriculares. Revista Iberoamericana de Educación Superior, 1(1), 37-57. Retrieved from 
http://ries.universia.net/index.php/ries/article/view/35/innovapdf

ECOSOC, C. E. y S. de las N. U. (2011). La visión de la UNESCO sobre el rol de las TIC en educación. In UNESCO (Ed.), Educación de calidad en la era digital. Una oportunidad de cooperación para UNESCO en América Latina y el Caribe (pp. 1923). Buenos Aires, Argentina: Oficina Regional de Educación para América Latina y el Caribe (OREAL-UNESCO).

ESPINOSA, J. (2010). Profesores y estudiantes en las Redes. Universidades Públicas y Tecnologías de la Información y la Comunicación (TIC). Ciudad de México: Juan Pablo Editor.

ESTEVE MON, F. M., LARRAZ RADA, V., GISBERT CERVERA, M., \& ESPUNY VIDAL, C. (2011). L'avaluació de la competència digital a través d'entorns de simulació 3D. In U. R. i Virgili (Ed.), Seminario Internacional Simul@. Tarragona.

GEORGE, D., \& MALLERY, P. (1995). SPSS for Windows. Step by step. Belmont (USA): Wadsworth Publising Company.

GUZMÁN FLORES, T. (2008). Las Tecnologías de la Información y la Comunicación en la Universidad Autónoma de Querétaro. Propuesta Estratégica para su Integración. Universitat Rovira i Virgili (Tarragona, España).

GUZMÁN FLORES, T., GARCÍA RAMÍREZ, M. T., CHAPARRO SÁNCHEZ, R., \& ESPUNY VIDAL, C. (2011). Formación docente para la integración de las TIC en la práctica educativa. Revista Apertura, 3(2), 6-13.

LÓPEZ de la MADRID, M. C. (2007). Uso de las TIC en la educación superior de México. Un estudio de caso. Apertura Impresa, 7, 63-81. Retrieved from http://www.udgvirtual.udg.mx/apertura/index.php/apertura4/article/view/94/10 5

LÓPEZ de la MADRID, M. C., \& CHÁVEZ ESPINOZA, J. A. (2013). La formación de profesores universitarios en la aplicación de las TIC. Sinéctica, (41), 2-18. Retrieved from http://www.scielo.org.mx/scielo.php?pid=S1665109X2013000200005\&script=sci_arttext\&tlng=pt\%5Cnhttp://www.scielo.org.mx/ pdf/sine/n41/n41a5.pdf

MAQUILÓN SÁNCHEZ JAVIER J., MIRETE RUIZ, A. B., GARCÍA SÁNCHEZ, F. A., \& HERNÁNDEZ PINA, F. (2013). Valoración de las TIC por los estudiantes universitarios ysu relación con los enfoques de aprendizaje. Revista de Investigación Educativa, 31(2), 537-554. http://doi.org/10.6018/rie.31.2.151891

MARTÍNEZ RODRÍGUEZ, F., \& GONZÁLEZ MARTíNEZ, J. (2015). Uso y apropiación de las Tecnologías de la Información y la Comunicación por parte de los docentes en las facultades de ingeniería. Redes de Ingeniería, 6(1), 6-24. http://doi.org/http://dx.doi.org/10.14483/udistrital.jour.redes.2015.1.a01

MEN, M. de E. N. (2008). Ruta de apropiación digital de TIC en el desarrollo profesional docente. Bogotá (Colombia).

MIRETE RUIZ, A. B. (2016). El profesorado universitario y las TIC. Análisis de su 
competencia digital. ENSAYOS. Revista de La Facultad de Educación de Albacete, 31(1), 133-147. Retrieved from https://revista.uclm.es/index.php/ensayos/article/view/1033

MORALES CAPILLA, M., TRUJILLO TORRES, J. M., \& RASO SÁNCHEZ, F. (2015). Percepciones acerca de la integración de las TIC en el proceso de EnseñanzaAprendizaje de la universidad. Pixel-Bit Revista de Medios Y Educacion, 46, 103117. http://doi.org/10.12795/pixelbit.2015.i46.07

POLANÍA-MUÑOZ, J. T., CÓRDOBA-BELTRÁN, M. A., \& LÓPEZ de PARRA, L. (2015). Uso de las TIC por parte de los profesores. Estado del arte (2009-2015). Revista FACCEA, 5(1), 84-93.

PRENDES ESPINOSA, M. . P., \& CASTAÑEDA QUINTERO, L. (2010). Universidades Latinoamericanas ante el reto de las TIC: Demandas de alfabetización tecnológica para la docencia. In J. M. Pérez Tornero (Ed.), Alfabetización mediática y culturas digitales (p. 17). Sevilla: Gabinete de Comunicación y Educación. Retrieved from http://www.gabinetecomunicacionyeducacion.com/es/publicaciones/comunicaci ones-alfabetizacion-mediatica-y-culturas-digitales

ROSARIO NOGUERA, H. J., \& VÁSQUEZ MELO, L. F. (2012). Formación del docente universitario en el uso de las TIC. Caso universidades públicas privadas. Pixel-Bit: Revista de Medios Y Educación, (41), 163-171. Retrieved from http://dialnet.unirioja.es/servlet/articulo?codigo=4097064\&info=resumen\&idiom $a=E N G$

SAID-HUNG, E., IRIARTE DÍAZ-GRANADOS, F., JABBA MOLINARES, D., RICARDO BARRETO, C., BALLESTEROS, B., VERGARA, E., \& ORDÓÑEZ, M. (2015). Fortalecimiento pedagógico en las universidades en Colombia a través de las TIC. Caso región Caribe. Educacion XX1, 18(2), 277-304. http://doi.org/10.5944/educXX1.14019

SÁNCHEZ, D., HOYOS, M., \& MEJÍA, M. (2015). Comportamiento macroeconómico del sector TIC en Colombia. Panorama TIC (Vol. XXXIII). Retrieved from http://www.americanbanker.com/issues/179_124/which-city-is-the-next-bigfintech-hub-new-york-stakes-its-claim-1068345-

1.html\%5Cnhttp://www.ncbi.nlm.nih.gov/pubmed/15003161\%5Cnhttp://cid.oxfo rdjournals.org/lookup/doi/10.1093/cid/cir991\%5Cnhttp://www.scielo

SANTAMARÍA, M., SANMARTíN, S., \& LÓPEZ, B. (2014). Perfiles de alumnos según el uso deseado de las TIC por el profesorado universitario. Pixel-Bit Revista de $\begin{array}{llll}\text { Medios } Y & \text { Educacion, 45, 37-50. }\end{array}$ http://doi.org/.doi.org/10.12795/pixelbit.2014.i45.03

SUÁREZ RODRÍGUEZ, J. M., ALMERICH, G., GARGALLO LÓPEZ, B., \& ALIAGA, F. M. (2010). Las competencias en TIC del profesorado y su relación con el uso de los recursos tecnológicos. Archivos Analíticos de Políticas Educativas, 18(10), 1-33. Retrieved from https://www.google.com.co/?gfe_rd=cr\&ei=u3hdU6nfE$r P 8 g f Q 84 D w B w \# q=$ analisis +discriminante+de+aplicacion+de+las+Tic+en+la+en se 2 anza \&start $=20$ 
TORRES GASTELÚ, C. A. (2011). Uso de las TIC en un programa educativo de la Universidad Veracruzana, Mexico. Actualidades Investigativas En Educación, 11(4), 1-22.

UNESCO. (2005). Formación docente y Tecnologías de la Información y la Comunicación. Experiencia de formación docente utilizando TIC. Santiago de Chile.

UNESCO. (2008). Estándares de competencia TIC para docentes. París.

VALERIO MATEOS, C., \& PAREDES LABRA, J. (2008). Evaluación del uso y manejo de las tecnologías de la información y la comunicación en los docentes universitarios. Un caso mexicano. RELATEC. Revista Latinoamericana de Tecnología Educativa, 7(1), 13-32. Retrieved from http://dialnet.unirioja.es/servlet/articulo?codigo=2859475

VERA NORIERA, J. Á., TORRES MORAN, L. E., \& MARTíNEZ GARCÍA, E. E. (2014). Evaluación de competencias básicas en tic en docentes de educación superior en México. Pixel-Bit: Revista de Medios Y Educación, 44, 143-155. Retrieved from http://dialnet.unirioja.es/servlet/articulo?codigo $=4532180$

VIVANCO, M. (2005). Muestreo estadístico. Diseño y aplicaciones. Santiago de Chile: Editorial Universitaria.

ZENTENO ANZIRA, A., \& MORTERA, F. J. (2011). Integración y apropiación de las TIC en los profesores y alumnos de educación media superior. Revista Apertura, (14). Retrieved from http://catedra.ruv.itesm.mx/handle/987654321/672

ZUBIETA, J., BAUTISTA, T., \& QUIJANO, Á. (2012). Aceptación de las TIC en la docencia. Ciudad de México: Universidad Nacional Autónoma de México.

\section{Para citar este artículo:}

González, J. \& Martínez, F. (2017). La percepción de los estudiantes acerca de la presencia de las TIC en la universidad. Un estudio en el ámbito de la Ingeniería en Colombia. EDUTEC, Revista Electrónica de Tecnología Educativa, 59. Recuperado el $\mathrm{dd} / \mathrm{mm} /$ aa de http://www.edutec.es/revista 